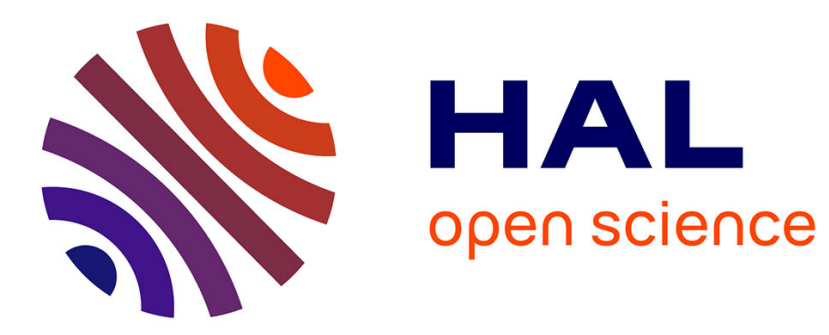

\title{
Electroencephalographic functional equivalence during observation of action
}

Paul Holmes, Dave Collins, Claire Calmels

\section{To cite this version:}

Paul Holmes, Dave Collins, Claire Calmels. Electroencephalographic functional equivalence during observation of action. Journal of Sports Sciences, 2006, 24 (6), pp.605-616. 10.1080/02640410500244507 . hal-01576398

\section{HAL Id: hal-01576398 https://hal-insep.archives-ouvertes.fr/hal-01576398}

Submitted on 5 Dec 2017

HAL is a multi-disciplinary open access archive for the deposit and dissemination of scientific research documents, whether they are published or not. The documents may come from teaching and research institutions in France or abroad, or from public or private research centers.
L'archive ouverte pluridisciplinaire HAL, est destinée au dépôt et à la diffusion de documents scientifiques de niveau recherche, publiés ou non, émanant des établissements d'enseignement et de recherche français ou étrangers, des laboratoires publics ou privés. 


\section{RJSP}

\begin{tabular}{|l|l|}
\hline Manuscript No. & A_124433 \\
\hline Author & \\
\hline Editor & \\
\hline Master & \\
\hline Publisher & \\
\hline
\end{tabular}

Journal of Sports Sciences

Typeset by

KnowledgeWorks Global Ltd.

for

Taylor \& Francis
Taylor \& Francis Group

\section{to be answered by AUTHOR}

AUTHOR: The following queries have arisen during the editing of your manuscript. Please answer the queries by marking the requisite corrections at the appropriate positions in the text.

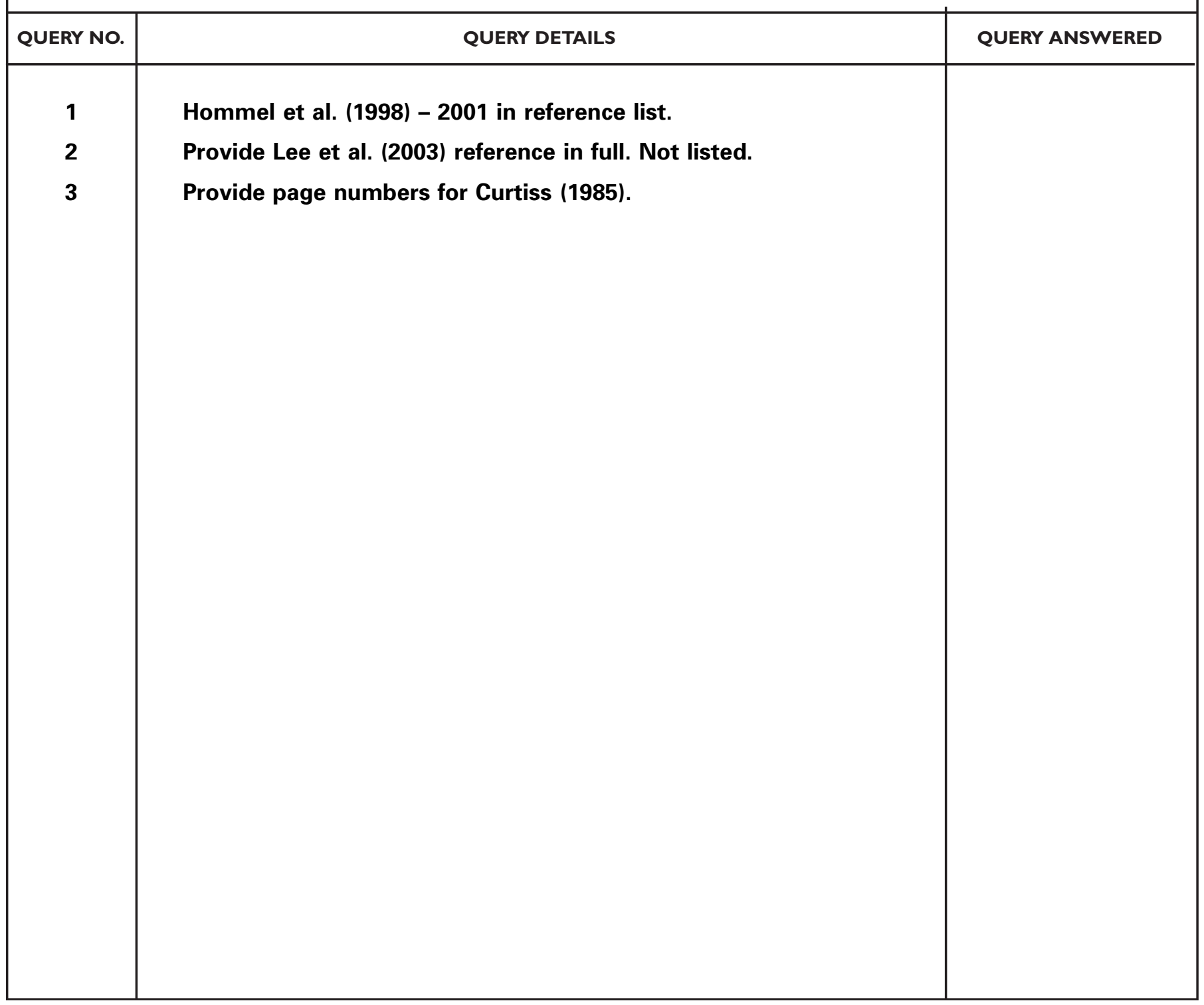




\title{
Electroencephalographic functional equivalence during observation of action
}

\author{
PAUL HOLMES $^{1}$, DAVE COLLINS $^{2}$, \& CLAIRE CALMELS ${ }^{3}$ \\ ${ }^{1}$ RIHSC: Biomedical, Health and Behavioural Sciences, The Manchester Metropolitan University, Alsager, UK, ${ }^{2}$ Department \\ of Physical Education and Sport, University of Edinburgh, Edinburgh, UK, and ${ }^{3}$ Institut National du Sport et de l'Education \\ Physique, Paris, France
}

(Accepted 4 fuly 2005)

\begin{abstract}
The purpose of this study was to examine the variability in cortical activation during physical air-rifle shooting and three different observation conditions. Elite air-rifle shooters performed a 40 shot individual match. Electroencephalograms were recorded from 11 active sites across the cortex during the final $6 \mathrm{~s}(3 \times 2 \mathrm{~s}$ epochs $)$ before shot release. Data collection was repeated while shooters watched a large-screen video of their worst shot performance from an internal-visual perspective when seated, standing and standing holding their rifle. The hypothesized differences between the three observation conditions and the physical shooting profile were not shown except at the left anterior temporal site, T5. This finding suggests that observation of performance cannot be differentiated clearly by posture or modified through kinetic and haptic afference, and that visual percepts predominate in observational functional equivalence. However, more functionally equivalent observation reduced the observation/execution variability over the temporal areas. Performer debriefs also identified different perceptions of physiological, psychological and behavioural functional equivalence associated with the different observation conditions. We conclude that elite performers' brains are accessed equally effectively during different observation conditions irrespective of some of the physical factors ascribed to the conditions. However, they may require more functionally matched conditions to attain greater equivalence in temporal areas.
\end{abstract}

Keywords: $E E G$, observation, functional equivalence, shooting

\section{Introduction}

Recent evidence has shown that similar representations are activated during perception and action

(1) (Hommel, Musseler, Aschersleben, \& Prinz, 1998; Prinz, 1997; for a review, see Grèzes \& Decety, 2001). Specifically, the common coding principle (Prinz, 1997) and motor-simulation theory (Jeannerod, 2001) presume that perceived events and planned actions fulfil two different functions, but that they share comparable neural networks. Similar neural processes have also been shown to be involved in the physical execution and observation of an action, since each operation is proposed to be assigned to the same internal brain representation (Grèzes \& Decety, 2001; Jeannerod, 1994).

Specific neurons have been discovered in the ventral premotor cortex of the macaque monkey (area F5). These neurons possess the ability to fire when the monkey executes a goal-directed hand movement (Rizzolatti et al., 1988) and also when it observes this same action executed by another monkey or by a human (Gallese, Fadiga, Fogassi, \& Rizzolatti, 1996; Rizzolatti, Fadiga, Gallese, \& Fogassi, 1996). These results offer the proposal for an execution/observation matching system. The neurons involved have been termed "mirror neurons", since their visual properties match, or mirror, their motor ones. Evidence in favour of the existence of such a system in humans has been provided by Fadiga, Fogassi, Pavasi, \& Rizzolatti (1995). They found that the muscular response pattern generated by a transcranial magnetic stimulus during an observation sequence was the same as that recorded while the participants executed the observed action. Similarly, Iacoboni, Woods, Brass, Bekkering and Mazziotta (1999), using functional magnetic resonance imaging, and Grèzes, Costes and Decety (1998), using positron emission tomography, have also verified the structural neuronal equivalence between observation and action in humans.

Correspondence: P. Holmes, Biomedical, Health and Behavioural Sciences, The Manchester Metropolitan University, Hassall Road, Alsager ST7 2HL, UK. E-mail: p.s.holmes@mmu.ac.uk 
Cochin, Bathelemy, Roux and Martineau (1998) examined neuronal activity while participants executed a pincer movement with the thumb and forefinger and then observed the same action. The authors found the existence of a similar oscillatory phenomenon in a section of the alpha frequency band $(7.5-10.5 \mathrm{~Hz})$ with alpha rhythm being blocked at nine scalp positions across the cortex. Similarly, Babiloni et al. (2002), using a self-initiated finger extension task, provided electroencephalographic data that support a functional equivalence (i.e. similar desynchronization and synchronization values in magnitude and timing) of alpha rhythmicity in premotor and primary sensorimotor cortex. They did not find functional equivalence in parietooccipital scalp regions, which suggests caution in making generalizations about the full extent of functional equivalence in observation conditions.

It would appear that functional equivalence - in this case, the extent to which observational neural correlates share mechanisms with the physical task may not be as clear as has been reported. For example, Decety et al. (1997) showed that, during action observation, different patterns of brain activity were elicited. The varying patterns were dependent upon the nature of the required executive processing (modified by the instructions given during the observation) and on the nature of the extrinsic properties of the action (whether they were meaningless or meaningful). The observation of meaningful, familiar actions involved the left hemisphere and, more specifically, the areas that coincide with the ventral visual pathway. In contrast, the observation of meaningless, unfamiliar actions engaged the right hemisphere and the areas associated with the dorsal visual pathway (Jeannerod, 1999). In addition, observation of an action with the aim of a later imitation involved the supplementary motor area, the ventral premotor cortex, and the dorsolateral prefrontal cortex, whereas observation of an action with the aim of a later recognition activated the temporal lobe and specifically the parahippocampal gyrus (Decety et al., 1997). It is clear that observational functional equivalence, and hence motor representation access, can be significantly influenced by task requirements. This equivalence remains to be tested in a sporting context.

\section{Electroencephalography and observation}

As discussed above, Cochin et al. (1998) have used the electroencephalogram (EEG) to consider mirror neuron activity in observation conditions with the event-related desynchronization and synchronization considered to reflect changes in parameters that control oscillations in neuronal networks and local interactions between main neurons and interneurons
(Pfurtscheller \& Lopes da Silva, 1999). Since EEG has also been used in psychophysiological studies of self-paced target sports (e.g. Konttinen \& Lyytinen, 1992; Loze, Collins, \& Holmes, 2001), analysis of background EEG activity profiles offers a well-tested approach for consideration of observation and action in rifle shooting. Previous studies have revealed that successful rifle and pistol shooting performance is associated with increasing alpha-band $(8-13 \mathrm{~Hz})$ power at certain sites across the cortex, especially the left anterior temporal cortex (e.g. Hatfield, Landers, \& Ray, 1984). In contrast, poor shots have been associated with event-related desynchronization (e.g. Loze et al., 2001).

Poor performance in rifle shooting has been shown to be a function of an inability to achieve, and maintain, a state of alert immobility and attentional preparedness (Era, Konttinen, Mehto, Saarela, \& Lyytinen, 1996). With decreasing visual feedback, shooters standing perpendicular to the target have a tendency to sway fore and aft with the additional weight of the gun. The task of sustaining a controlled and stable bipedal stance is particularly demanding because the centre of gravity is raised and moved anteriorly through the inclusion of a rifle weighing approximately $7 \mathrm{~kg}$. Consequently, to aid postural stability, shooters adopt a specific stance to isolate trunk and lower limb movement and wear specially manufactured, stiffened leather and canvas jackets and trousers and a padded glove on the nontriggering hand. Concave-soled boots are also worn to improve balance and provide ankle support. The equipment is proposed to provide a $30-70 \%$ benefit to postural stability (Era et al., 1996). However, Era and colleagues have also demonstrated that, irrespective of shooting clothing, competitive shooters are better able to stabilize their postural control than naive shooters. The extent to which these different percepts may contribute to effective functional equivalence during observation of shooting has not been considered. Thus, perception of self-action in a standing position, while wearing appropriate clothing, may result in a greater similarity of brain activity as compared to a sitting position in non-sporting clothes.

\section{Aims of the study}

Janelle et al. (2000) and Loze et al. (2001) have provided evidence that supports the existence of an individualized brain motor representation system that is accessed differentially for shot quality. The arguments from functional equivalence research (e.g. Holmes \& Collins, 2001) suggest that modification towards, and inclusion of elements from, the physical task in any observation-based procedure will access greater proportions of the motor representation associated with the behaviour; increased covariance 
is displayed through numerous psychophysiological and behavioural indices. If this is the case, then sport psychologists with responsibility for mental training through the use of, for example, video will need to consider carefully all possible parameters that may impact upon observational functional equivalence if they are to use the tool to effectively reinforce the brain representation for the skill. Brain activity during observation of a video may be more similar to the physical condition if the performer watches while holding a piece of relevant sporting equipment, such as a rifle. This additional afferent information will be available for representational access.

Given this information and the shooter's perceived reliance on his or her shooting position and clothes, this study aimed to determine the extent to which these prerequisites for success in physical shooting contribute to the electroencephalographic functional equivalence of the skill during observation conditions. Specifically, the study aimed to: identify EEG alphaband power topographies during different observation modalities and consider qualitative comments of perceived preference; and examine the functional equivalence of different observation modalities.

It was hypothesized that modifying the observation conditions would significantly alter the EEG alpha synchronization and desynchronization profile. This would be achieved by introducing more relevant postural forces and more relevant kinetic and haptic afference from the rifle and trigger. It was also hypothesized that EEG functional equivalence would increase across all electrode sites as elements of the physical shooting condition were increasingly introduced.

\section{Methods \\ Participants}

The participants were six nationally ranked female air-rifle shooters comprising the full membership of a National Rifle Squad competing in the disciplines of air-rifle, three-position rifle and prone rifle. All the shooters had considerable international experience at senior and junior level. They were aged $18-36$ years (mean age $=27.0, s=5.3$ years) and had shooting training experience of between 5 and 25 years (mean training experience $=14.8, s=6.3$ years). Employing elite participants from rifle shooting ensured that the assessment environment offered a consistent task difficulty approached with significant goal-directed behaviour. The air-rifle match personal best scores ranged from 384 to 396 out of a maximum 400 (mean personal best $=389.8, s=4.12$ ). In addition, all participants were familiar with the use of video as a training aid. Before the test sessions, participants completed the Edinburgh Handedness Inventory
(Oldfield, 1971) to provide an index of laterality, since handedness may affect patterns of cerebral lateralization (Curtiss, 1985). All participants were scored as right-hand dominant. Using simple, wellestablished aiming tasks, National Squad coaches established eye dominance. These confirmed that the right eye was dominant for all shooters. The local institutional ethics committee approved the study's procedures and written informed consent was provided by all shooters before participation.

\section{Experimental procedure}

All testing took place in a specially constructed shooting range in our laboratories developed under the guidance of the national governing body for the sport. Shooters habituated to the environmental light conditions and went through their normal time-scale competition preparation procedures. The target was lit with light of 1000 lux. On all occasions, the shooter's personal coach was present and was allowed to interact with the shooter in the same way as they would before normal competition. Data were collected during physical shooting performance and in three different observation conditions.

A video camera, situated directly over the shooter's right shoulder, provided a picture containing views along the barrel of the gun and a distant picture of the target. This was used to provide an internal-visual perspective video for the observation conditions.

\section{Performance assessment}

Shots were scored using the SCATT optical shooting system (ZAO SCATT, Zelanograd, Russia), since electronic scoring is a common feature of elite air-rifle shooting. The SCATT system was calibrated for each shooter to ensure the computer-generated target directly reflected the paper target and, therefore, provided highly accurate scoring $(0.5 \pm 0.1 \mathrm{~mm})$. The equipment set-up described was familiar to all shooters, since it formed a regular part of their daily training programme. Post-match debriefs confirmed little or no disruption to their normal shooting behaviour. All shooters were experienced in reporting perceived shot quality and "called" the shot immediately after triggering and before any feedback from the coach, SCATT system or actual targets.

The multiple assessment procedure, combined with post-hoc, coach-directed video analysis, provided a highly valid shot quality assessment and separation of successful and unsuccessful shots. Emphasis was placed on the quality of the shot trace-length provided by the SCATT system, recognized as a critical marker of elite shooting quality (Flippant \& Levene, 1998). Because of the sensitivity of the SCATT system, pre-triggering movement, triggering 
errors and trigger "snatches" were easily identified. The occurrences of these, and other coach-identified rifle performance errors, were used as markers of poor shot quality. Loze et al. (2001) have shown that EEG more clearly differentiates poor shots, in contrast to good shots, from the average shot profile. This may be because poor shots are rare and associated with more emotion than positive behaviours (Lang, 1979). The EEG profile of the worst shot was used to represent the physical trial for each shooter and selected for use in the observation trials. As discussed above, Loze et al. (2001) have shown that poor shots appear to be associated with greater alpha-band desynchronization over occipital and temporal sites in contrast to the robust finding of increased alpha-band power (synchronization) for good performances.

Therefore, each shooter's worst shot was recorded as the physical trial and used as the comparison trial in all subsequent observation conditions. This approach was only used for the purpose of the study and all shooters were made aware of this protocol through the written informed consent form. All shooters were provided with training videos highlighting successful shots for subsequent mental skills training purposes.

Visual percepts may not be the only criteria to modify functional equivalence. Therefore, aspects of force (Decety, Jeannerod, \& Prablanc, 1989) and haptic afference (Beisteiner, Höllinger, Lindinger, Lang, \& Berthoz, 1995) were also included as potential interacting independent variables within the observation conditions. These factors were manipulated through sitting and standing, and with and without gun hold. Additional force and afference produced by the shooter's suit remained constant throughout all observation conditions. Participants were tested in randomized observation conditions and were fully debriefed through an inductive interview process after the final condition as a manipulation check for the observation process and to obtain qualitative comments on their experiences in each condition. The interview probed themes relating to psychophysiological correlates of shooting performance, functional equivalence and mirror neuron activity. These included: attentional focus; conscious awareness of the pre-shot routine and the shot; self-talk; technical awareness; visual perspective; and physiological functional equivalence.

The three observation conditions are now described in detail.

Observation condition 1. Participants sat on a normal office-type chair with a trunk/hip angle and knee angle of $90^{\circ}$. Hands were placed pronated on the thigh with no rifle visually present. A microphone, used to identify the shot in the physical condition, was placed next to the video monitor to digitally mark the shot on the EEG data capture, thus allowing the prior $6 \mathrm{~s}$ period to be identified. The video monitor was sufficiently large, and at an appropriate distance, to reduce any potential eye movements as a result of picture scanning. Participants watched the video of the bad shot filmed from the internal-visual perspective and were provided with no instructions other than that they should watch the shot as if they were shooting it. This procedure was repeated four times, with the instructions being provided before each EEG data capture.

Observation condition 2. In this condition, participants stood in a position similar to the anatomical standing position. They watched the bad shot and were provided with the instructions previously mentioned before each shot/data collection.

Observation condition 3. In this condition, the participants were again required to stand to conduct the session. In addition, their rifle was introduced and participants were asked to take up their normal shot position before the video was played. The same verbal instructions were provided before each data capture.

\section{Electroencephalographic recording}

The alpha rhythm offers a useful non-invasive and temporally fine-grained measure of regional hemispheric activation (Davidson, 1988), and has been successfully used in previous studies of elite shooting performance (e.g. Bird, 1987; Hatfield et al., 1984; Loze et al., 2001).

Cephalic activity was assessed at 11 sites: F3, F4, T3, modified C3, modified C4 ('C3, 'C4), T4, T5, $\mathrm{P} 3, \mathrm{P} 4, \mathrm{~T} 6$, and $\mathrm{Oz}$ with a common average reference (Cooper, Osselton, \& Shaw, 1974) located in accordance with the International 10-20 system (Jasper, 1958). The central sites were modified by positioning the electrode $1 \mathrm{~cm}$ anterior of the standard site to record activity over the premotor cortex. The ground electrode was positioned on the forehead. The EEG data collection followed the tested method of Loze, Collins and Shaw (1999). Electrode sites were prepared with Omni prep skin preparation paste (D. O. Weaver and Co., Aurora, USA) and $8 \mathrm{~mm} \mathrm{Ag} /$ $\mathrm{AgCl}$ electrodes were attached with Collodion Adhesive (SLE Diagnostics, Croydon, UK) because of the potential heating effects on the head as a result of the leather and canvas suits worn by the rifle shooters. Dracard electrode gel (Dracard, London, UK) was used as the conductive fluid and injected into the electrode cups. All sites were allowed to settle for $15 \mathrm{~min}$ before impedances were checked (Picton \& Hillyard, 1972; Regan, 1989). Impedance was kept homogeneously below $5 \mathrm{k} \Omega$. Amplifier bandwidth 
was set between 0.5 and $70 \mathrm{~Hz}$. Activity was sampled at $550 \mathrm{~Hz}$, with a gain of 30,000. Artefact-free EEG data were collected for $10 \mathrm{~s}$.

Electro-oculogram (EOG) correction was not possible for noise reduction, since the shooters commented, during pilot testing, that the EOG electrodes interfered with their shooting glasses and/or provided a general "discomfort" during aiming. However, the sport, by its very nature, is characterized by extreme eye stillness with extended periods of no blinking as the shooter attempts to aim. This is supported by the findings of Vickers (1996), who suggested that elite performers extend eye fixations when aiming at targets similar to the $10 \mathrm{~m}$ distance used in air-rifle shooting.

The EEG montage described above was maintained for the observation EEG data acquisition sessions. All electrodes were checked for drift in impedance before each data collection period.

\section{Data processing}

The EEG data were time-locked to trigger release through a unidirectional microphone positioned adjacent to the gun barrel exit identifying shot release (T0). Shots were also manually marked and coded through the computer keyboard.

The EEG data were captured throughout the shooting match and stored in computer memory. Data were subsequently reduced to a $6 \mathrm{~s}$ preparation phase (T-6) prior to T0, which was divided into $3 \times 2$ s EEG epochs. Previous research (e.g. Konttinen, Lyttinen, \& Viitasalo, 1998; Loze et al., 2001) has identified that significant changes occur in the EEG in the final seconds of shot preparation and that $2 \mathrm{~s}$ epochs are appropriate for observing the temporal nature of such changes.

Displays of digitally converted EEG data were visually inspected off-line, to eliminate EEG segments contaminated with ocular or muscular artefacts from the shot preparation epochs. Post-hoc video and EEG analysis did not eliminate any shots for reasons of eye blink, eye movement or muscle activity. A raised cosine window was applied before the 256 block size Fast Fourier transform (Cooley \& Tukey, 1965) calculations. Spectral analysis yielded absolute power values $\left(\mu \mathrm{V}^{2}\right)$ for the alpha-band frequency range $(8-13 \mathrm{~Hz})$ for each of the three epochs before the shot.

Since participants observed their worst shot in three situations for four trials, a greater number of trials for each observation condition may have induced a modified profile of activity. There is evidence that repeated exposure to the same stimulus can significantly modify the EEG profile of the

(2) participant (Lee et al., 2003). Repeated presentation of the same stimulus caused a gradual reduction of cephalic activity. It is likely, therefore, that if we had increased the number of exposures in each observation condition, the profile of activity would have represented a significantly lower version of activity due to the number of observation trials undertaken by the participants. The time-scale of such decay remains to be tested. In addition, there is increasing evidence that single-trial EEG is a valid technique. Evidence presented by Delorme and Makeig (2004) provides strong evidence for single-trial EEG dynamics. Similarly, Delorme, Makeig, Fabre-Thorpe and Sejnowski (2002) have shown reproducible patterns of activation at several brain locations for one participant across two sessions. They also reported that such techniques can reveal the dynamics of brain activation and synchronization phenomena that are not revealed by standard averaging methods. Traditional EEG assessment typically involves the averaging of several electroencephaolgrams ( $n>30$; Pfurtscheller, 1999) for the explicit purpose of yielding stable patterns of activity. As discussed above, we did not feel it was possible to use this approach with this design.

\section{Statistical analyses}

An analysis of variance with repeated measures was calculated for the two within-subject factors, using condition (physical condition, observation condition 1 , observation condition 2 , observation condition 3 ) as the first factor and epoch (epoch 1, epoch 2, epoch 3) as the second factor. Bonferroni corrections were made to all alpha levels $(P<0.0167)$ to control for Type-I errors. Post-hoc comparisons were calculated using Tukey's HSD test. To monitor the meaningfulness of the observed differences, measures of effect size were computed using pooled standard deviations (Hedges \& Olkin, 1985).

\section{Results}

Statistical analyses only yielded significant main effects for the Epoch factor for the site T6 $\left(F_{2,10}=\right.$ $12.658, P=0.002)$. No main effect was found for the Condition factor at any site. A significant Conditio$\mathrm{n} \times$ Epoch interaction was only observed for electrode site T5 $\left(F_{6,30}=3.339, P=0.015\right)$. For electrode site $\mathrm{T} 5$, post-hoc tests revealed that in epoch 1, alpha-band power values were significantly higher in the physical condition (40.07) than in observation condition 3 (22.77) $(P=0.0028$, effect size $=0.06)$. In the physical condition, alpha-band power was also higher in epoch 1 (40.07) than in epoch 3 (23.94) $(P=0.0063$, effect size $=0.05)$. Descriptive statistics for the 11 active sites and the four conditions are provided in Table I. Graphical 


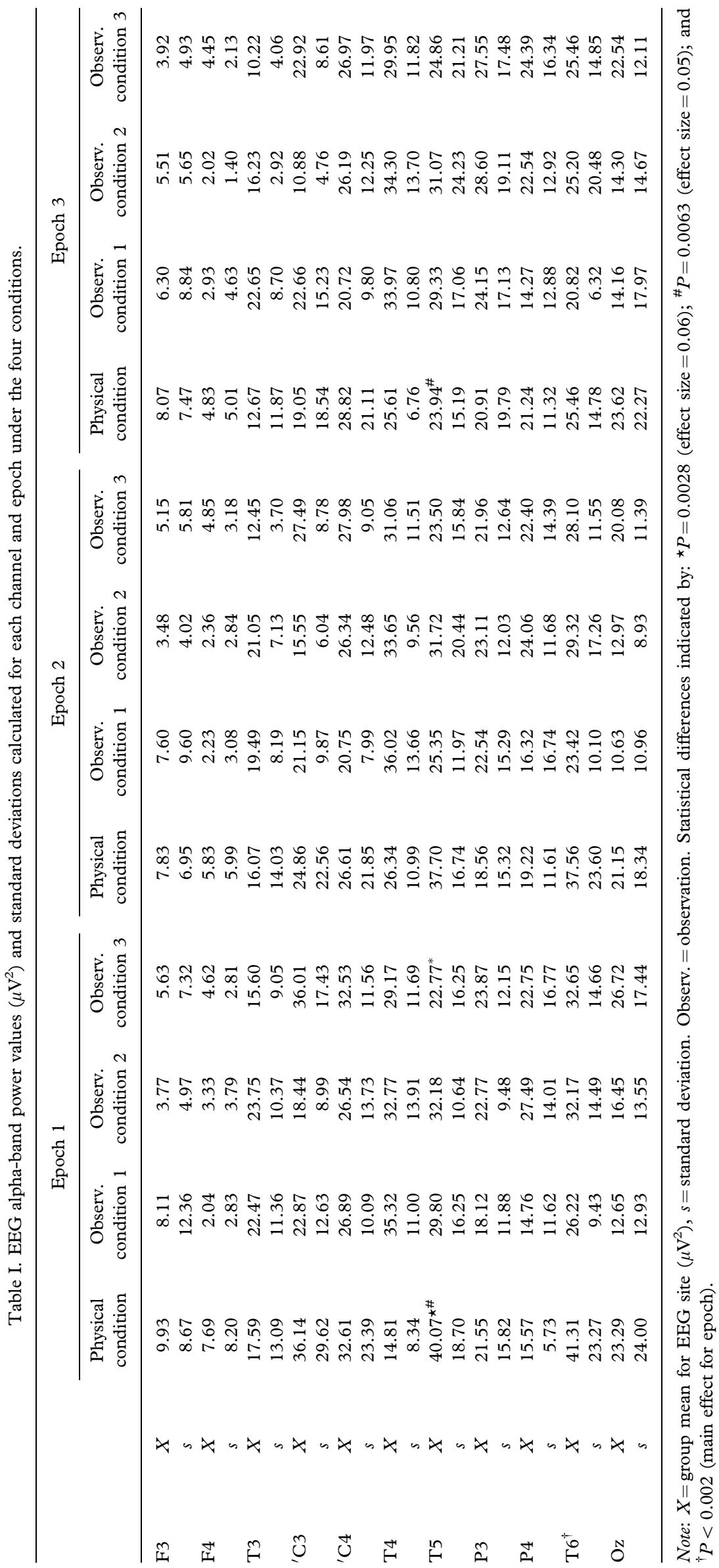


interpretations of the EEG data for all 11 sites can be seen in Figure 1.

\section{Discussion}

The present study was designed to consider the electroencephalographic profiles of the physical preparation and execution of three different observation conditions of elite air-rifle shooting. Low levels of alpha-band activity occurred across the three epochs. In many cases, we observed increasing desynchronization from epoch 1 to epoch 3. The physical EEG profile was highly reproducible in all three observation conditions. We acknowledge that EEG analysis through power spectrum analysis can only ever provide a precise indication of cortical events and that the technique can only reveal part of the relevant processing, as it is used as an index of local cortical engagement. However, using this approach will allow practitioners to make useful comparisons with earlier research that has also used this analysis technique (e.g. Kerick et al., 2001). We would encourage future studies of EEG in sport to consider power, coherence (Deeny, Hillman, Janelle, \& Hatfield, 2003; Serrien, Fisher, \& Brown, 2003) and synchronization likelihood (Stam \& van Dijk, 2002) to gain a more detailed understanding of cortico-cortical communication during sporting behaviour.

\section{Observation of shooting conditions}

One of the main concerns for the present study was to ensure the validity of the physical EEG profile to allow comparison of the observation conditions. A number of markers, consistent with previous research, suggest this was achieved: the repetition of the robust findings from the left anterior-temporal sites where alpha-band power desynchronized (e.g. Loze et al., 2001); the temporal profile asymmetry (T3:T4); and the increasing desynchronization at 'C3 (again, in contrast to the profile at ' $\mathrm{C} 4$ ).

The environmental conditions and the EEG assessment techniques provided data that are consistent with the physical EEG data of previous studies which identified increased or maintained cortical processing during the pre-shot period of poor performance in target-based sports (e.g. Bird, 1987; Hatfield et al., 1984; Loze et al., 2001).

The EEG data provide considerable support for the concept of a motor representation accessed by both motor preparation/execution and observation. Electroencephalography was able to identify the topographic detail of the observation conditions, but the data offer only partial support for the hypothesis that modification of the observation modality affects the extent of the functional equivalence.
Functional equivalence was shown through two primary correlates. First, the observation profiles spatially mapped onto the physical preparation and execution profile. Second, direct quantitative matching of EEG activity at a given epoch was found. Both of these correlates support the work of Babiloni et al. (2002) and both forms of functional equivalence would be expected to be present to sustain the contention of a fully shared representation. However, as Decety and Sommerville (2003) have argued, sharedness does not mean identicality; otherwise representations would completely overlap and lead to behavioural and agency confusion. Babiloni et al. (2002) showed that functional equivalence was negligible in parietal-occipital scalp regions. This is a finding which the authors suggest could be the neural substrate to distinguish agency. While our findings reveal sites where the observation conditions showed no statistically significant difference to the physical condition, the data show that the neural substrate for observation was not identical to that for physical shooting. We therefore support Decety and Sommerville in that the term "functional equivalence" should be understood to mean similarity and not identicality of neuronal activity.

We hypothesized that there would be noticeable variation in functional equivalence between the different observation conditions and the physical profile. The execution/observation profile similarities were, therefore, surprising. Loze et al. (2001) and Kontinnen and Lyytinnen (1992) have argued that the critical time for elite shooters is during the final $2 \mathrm{~s}$ before trigger pull (epoch 3 in this study). At 10 of the 11 active sites during epoch 3, there was no statistically significant difference in alpha-band power between any of the four conditions. This finding suggests that the shooters were able to extract meaningful information from all the observation conditions. We propose here that the lack of significance between the three observation conditions is a function of the skill level of the performer. Their skill level offers a reinforced and fully integrated motor representation for the task. This line of argument would be supported by previous neuronal network-based concepts steeped in parallel, vertical and horizontal levels of processing. The video percepts seemed to have offered the shooters sufficient visual information to functionally access the motor representation irrespective of the observation condition, suggesting that the visual modality takes a primary role in observation conditions. Whether this is true to for the external visual perspective remains to be tested.

While strong support was offered for neuronal functional equivalence from all observation conditions, visual inspection of the data suggests that there 
T3

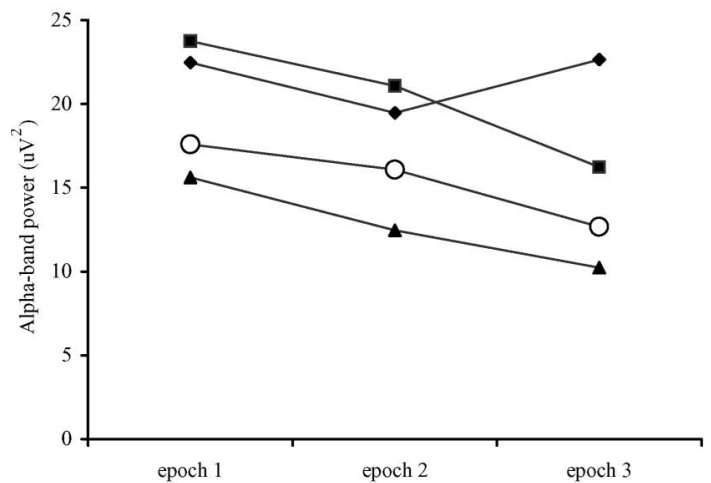

'C3

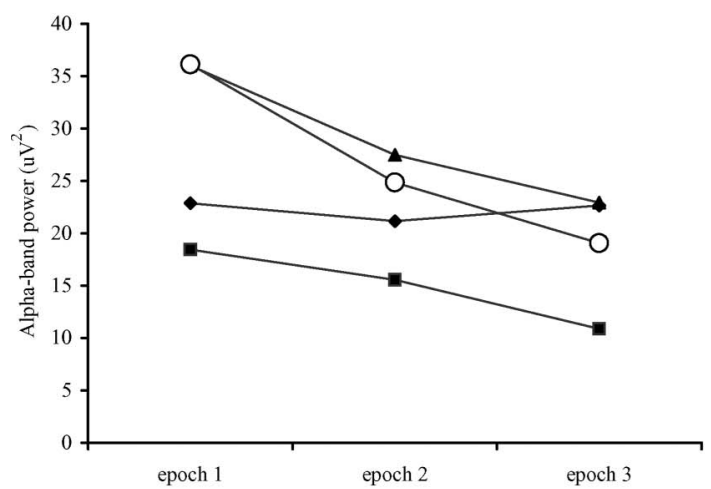

P3

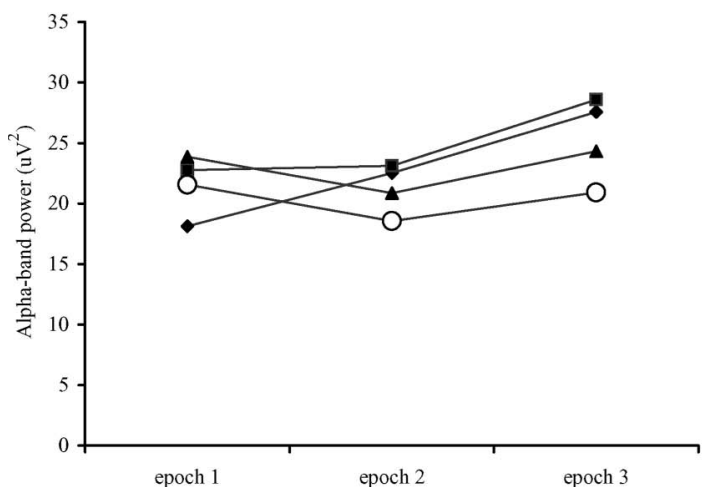

T4

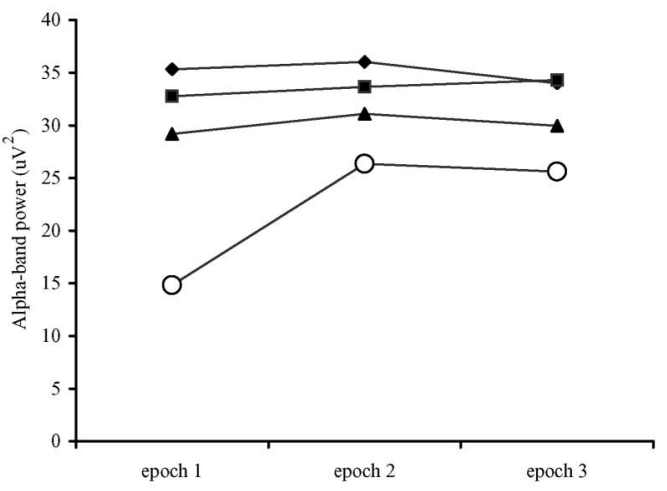

'C4

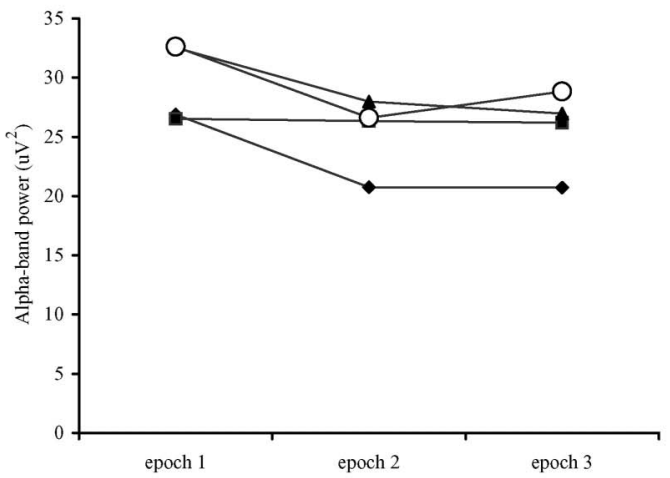

P4

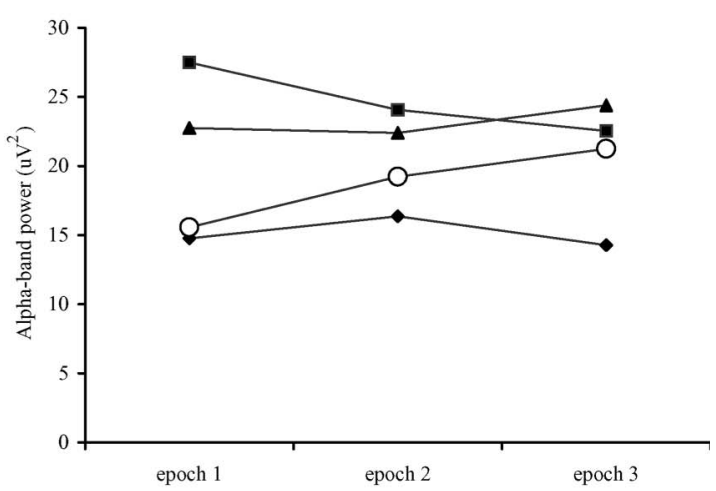


T5

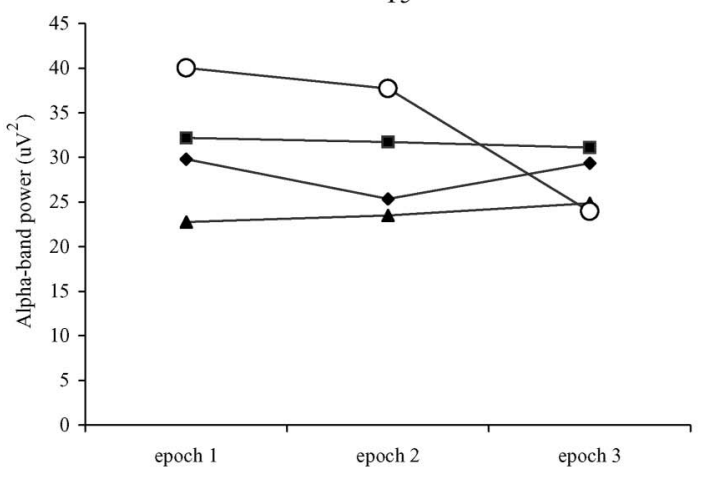

F3

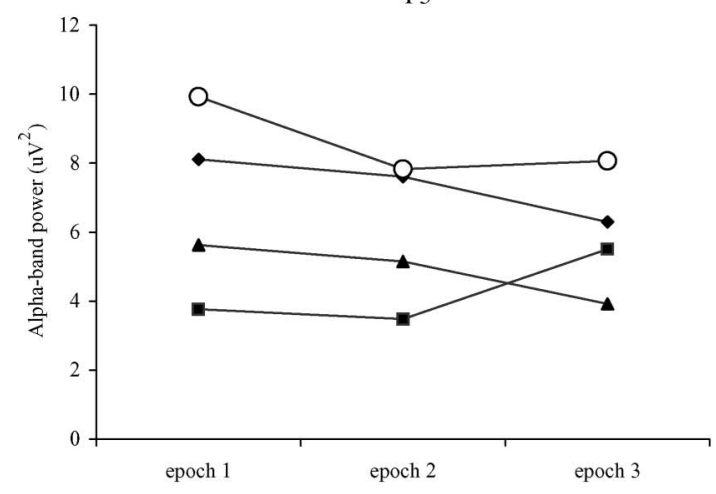

$\mathrm{Oz}$

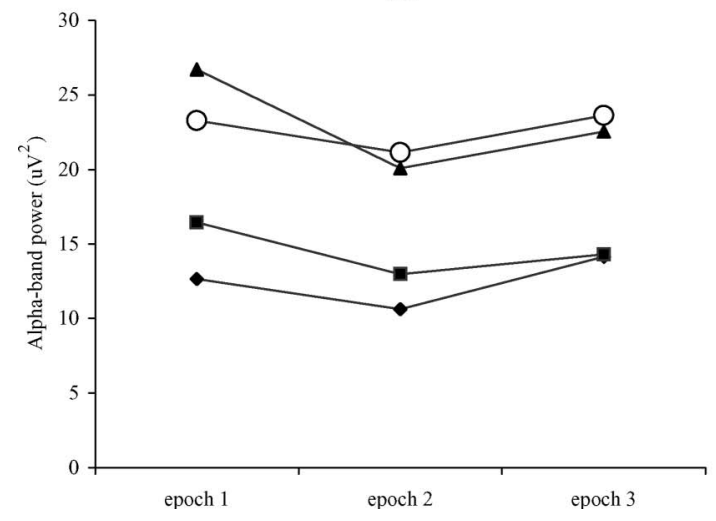

was evidence from some sites that observation condition 3 was the most successful. However, what was not clear was the contribution of sub-components of the observation modality to the overall functional equivalence. In condition 3 , there were many possible interacting factors that were deemed to contribute to the neuronal substrate. The primary factors were the relevant postural force, rifle force and tactile afference. It was hypothesized that they would contribute to functional equivalence in some simple linear fashion. This was not the case. There was some
T6

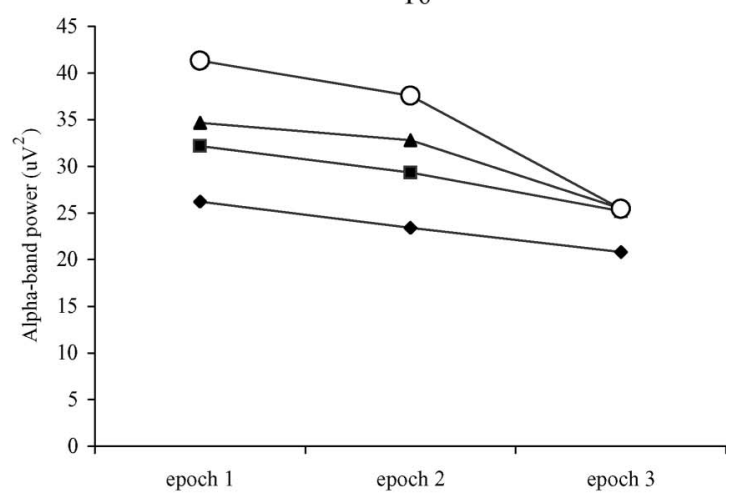

F4

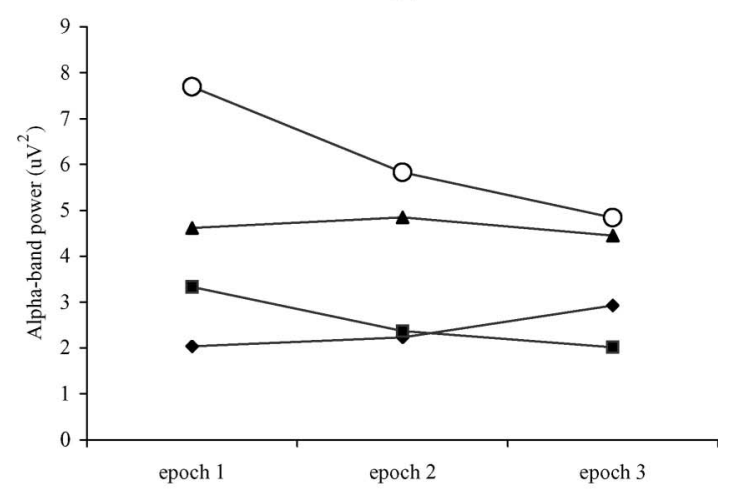

Figure 1. Averaged epochs of the group EEG alpha-band power at 11 sites for physical shooting and three observation conditions. The first epoch is an average of sample values at -6 to $-4 \mathrm{~s}$, the second at -3.999 to $-2 \mathrm{~s}$, and the third at -1.999 to 0 (T0) s. $\bullet$, observation condition $1 ; \mathbf{\square}$, observation condition 2 ; $\mathbf{\Delta}$, observation condition $3 ; 0$, physical condition.

evidence that the interaction of these three elements was more effective in increasing functional equivalence. It is possible that the interaction of all the components was the critical factor rather than any one component being the single main effect. Unfortunately, it is unlikely that alpha-band power studies will be able to discriminate the detail required to consider this issue more fully; the variance in the underlying activity being greater than the difference between any of the functional equivalence sub-components. There may be an optimal amount of functional equivalence 
after which any additional attempts to increase it merely serves to introduce noise into the system. Functional equivalence must, therefore, be considered within the Langian concept of the meaning proposition (Lang, 1979) and be appropriate for the performer.

The observation conditions were able to provide perceptual information to allow functional equivalence in visual cognition. Statistically, there was no difference between the three observation conditions, possibly because in all conditions the shooters watched the same internal perspective video. However, visually, Figure 1 shows that observation condition 3, across all sites, displays activity which is more closely aligned to the physical condition in terms of magnitude and timing. This suggests that, in addition to the visual information, the kinetic and haptic information may have had some relevance to the overall functional equivalence of the visual behaviour. This cautious proposal remains to be tested further.

The EEG activity over the anterior-temporal site during observation was particularly interesting. As mentioned earlier, one of the most robust findings in the shooting literature has been that of alpha desynchronization over this area during poor shooting (e.g. Hatfield et al., 1984; Loze et al., 2001). We found a similar typography in this study. The observation conditions suggest that posture may be an important factor in obtaining greater functional equivalence in the temporal areas. At the critical third epoch, observation condition 1 showed alpha-band power synchronization in contrast to desynchronization in both the other two observation conditions and the physical condition. The lack of the rifle in observation condition 2 did not appear to influence the extent of functional equivalence at T3.

The anterior-temporal area of the left hemisphere, including Broca's area, has been proposed as a key site for a mirror neuron system in humans (Fadiga et al., 1995). If, as the findings from this study appear to indicate, Broca's area is involved with action recognition, then as Hamzei et al. (2003) have proposed, it is tempting to speculate that language production and action recognition share a common functional architecture. We cannot comment on auditory processing, since the anatomical location of the auditory cortex is in the supratemporal plane and, as such, it is hard to detect with scalp-recorded EEG (Pfurtscheller \& Lopes da Silva, 1999). There is some evidence from the data at $\mathrm{T} 3$ and $\mathrm{T} 5$ that greater matching of behaviour is associated with greater EEG functional equivalence in the temporal areas. Mirror neuron activity during observation does seem to be influenced by kinetic and haptic afference.
Therefore, since the nature of the observation conditions in this study may be differentially meaningful to the shooters, we can provide some support for the claims of Decety et al. (1997) that different patterns of brain activity are elicited under different meaning conditions. Whether this pattern of activity would be retained for observation of good shots, where previous research has shown significant alpha synchronization, remains to be seen. While there is anecdotal evidence for the meaningfulness of different successful performances, the emotional congruence may be less elaborate than for poor performances (cf. Lang, 1979).

In addition, there was observable evidence that the shooters moved their trigger finger in both observation conditions 2 and 3 , but not condition 1 . In condition 3 , the shooter's trigger finger was resting on the trigger, in exactly the same way as in physical shooting; in observation condition 2, it moved as if it was pulling the trigger, even though the trigger was not present. This behaviour could account for the contralateral alpha-power desynchronization profiles seen in epochs 2 and 3 for observation in these conditions. Interestingly, however, in observation condition 1, relative synchronization was found from epoch 2 to epoch 3 . The central ' $\mathrm{C} 3$ profile seems to reflect contralateral triggering behaviour. While not statistically significant, the typology of the activity provides some support for the differential effects on central and behavioural functional equivalence in observation conditions 2 and 3 .

All shooters were involved in an independent, detailed debrief following the observation trials. Unlike the EEG data, the observation conditions were, according to the shooters' perceptions, differentiated by the kinetic, kinematic and haptic variables associated with them. These preferences may be an important consideration for coaches and sport psychologists using observation as an intervention. The following excerpts from two shooters highlight the perceived functional equivalence that was experienced:

when you gave me the gun, it made me feel like that was me now so I thought more of what I was doing 'cos it was like I was shooting now if you know what I mean so it was better than before, but yer know, worse 'cos it was a bad shot [laughs]... These standing ones will be better for mental prep. 'cos it was like real-time not like from the other angle [referred to side-on view]... it kinda sucked me into that person 'cos it felt like real- time... It felt more like I was shooting that one [nods head]. Yeh, so I was trying to stop myself saying don't shoot it, it was like I was shooting [pause]. It was really weird really ... my breathing was slowing and I was trying to tell 
myself to stop myself talking to myself like real shooting, like it was me, do you know what I mean ... I was more aware of what I was doing but I wasn't really aware of when the shot was going off... it really felt like me... These standing ones are better 'cos yer don't focus so much on the technical stuff like the coaching ones [external visual perspective] and it's easier to feel it when you're standing. (Shooter VE)

It's like I can feel the shot much more and it makes me like go through the same feelings of the bad shot like it was real ya know ... I knew I wasn't still on that one and this just proves it, but much more ya know, 'cos I can feel me swaying and triggering too much like I'm there ya know ... in that last one I was waiting for the snatch. (Shooter VT)

Within these example reports there is evidence of: changes of attentional focus, ("made me feel like that was me now"); more verbal and conscious attentional focus ("thought more of what I was doing", "more aware of what I was doing"); differences between visual perspectives ("not like from the other angle"); negative self-talk ("stop myself saying don't shoot it"); physiological functional equivalence ("my breathing was slowing"); and less conscious awareness of technical components of the skill with more functionally equivalent observation ("the standing ones are better 'cos you don't focus so much on the technical stuff', "it's easier to feel it when you're standing").

The reports of breathing rate slowing have also been observed during motor imagery (e.g. Williams, Rippon, Stone, \& Annett, 1995) and reflect the peripheral functional equivalence that can accompany imagery and, in this case, observation. More elaborate and equivalent profiles during observation have been inferred to reflect more functional representational access (e.g. Shaw, Holmes, Wilkerson, \& Jones, 2004).

The references to attentional changes, self-talk and technical awareness, considered alongside the EEG data, provide further evidence that practitioners should consider carefully aspects of posture and haptic afference when using video for observation.

The observation of action from an internal visual perspective offered an effective technique for accessing the motor representation of elite rifle shooters. The visual information and temporal equivalence contained within a video of human shooting behaviour allowed for preferential access to appropriate visuomotor cortical networks, especially as performance neared the goal of the action. Although the evidence for functional equivalence within the sporting context is at a relatively early stage, the data discussed here would seem to provide some support for the hypothesized links to the physical representation.

\section{References}

Babiloni, C., Babiloni, F., Carducci, F. et al. (2002). Human cortical electroencephalography (EEG) rhythms during the observation of simple aimless movements: A high resolution EEG study. NeuroImage, 17, 559-572.

Beisteiner, R., Höllinger, P., Lindinger, G., Lang, W., \& Berthoz, A. (1995). Mental representations of movements: Brain potentials associated with imagination of hand movements. Electroencephalography and Clinical Neurophysiology, 96, 183-193.

Bird, E. I. (1987). Psychophysiological processes during rifle shooting. International fournal of Sport Psychology, 18, 9-18.

Cochin, S., Bathelemy, C., Roux, S., \& Martineau, J. (1998). Observation and execution of movement: Similarities demonstrated by quantified electroencephalography. European fournal of Neurosciences, 11, 1839-1842.

Cooley, J. W., \& Tukey, J. W. (1965). An algorithm for the machine calculation of complex Fourier series. Mathematical Computing, 19, 297-301.

Cooper, R., Osselton, J. W., \& Shaw, J. C. (1974). EEG technology (2nd edn.). London: Butterworths.

Curtiss, S. (1985). The development of human cerebral lateralization. In D. F. Benson \& E. Zaidel (Eds.), The dual brain: Hemispheric specializations in humans (pp. 000-000). New York: Guilford Press.

Davidson, R. J. (1988). EEG measures of cerebral asymmetry: Conceptual and methodological issues. International fournal of Neurosciences, 39, $71-89$.

Decety, J., Grèzes, J., Costes, N. et al. (1997). Brain activity during observation actions: Influence of action content and subject's strategy. Brain, 120, 1763-1777.

Decety, J., Jeannerod, M., \& Prablanc, C. (1989). The timing of mentally represented actions. Behavioral Brain Research, 34, $35-42$.

Decety, J., \& Sommerville, J. A. (2003). Shared representations between self and other: A social cognitive neuroscience view. Trends in Cognitive Sciences, 7, 527-533.

Deeny, S. P., Hillman, C. H., Janelle, C. M., \& Hatfield, B. D. (2003). Cortico-cortical communication and superior performance in skilled marksmen: An EEG coherence analysis. Fournal of Sport and Exercise Psychology, 25, 188-204.

Delorme, A., \& Makeig, S. (2004). EEGLAB: An open source toolbox for analysis of single-trial EEG dynamics including independent component analysis. Fournal of Neuroscience Methods, 134, 9-21.

Delorme, A., Makeig, S., Fabre-Thorpe, M., \& Sejnowski, T. (2002). From single-trial EEG to brain area dynamics. Neurocomputing, 44/46, $1057-1064$.

Era, P., Konttinen, N., Mehto, P., Saarela, P., \& Lyytinen, H. (1996). Postural stability and skilled performance: A study of top level and naïve rifle shooters. Fournal of Biomechanics, 29, 301-306.

Fadiga, L., Fogassi, L., Pavasi, G., \& Rizzolatti, G. (1995). Motor facilitation during action observation: A magnetic stimulation study. Fournal of Neurophysiology, 73, 2608-2611.

Flippant, P., \& Levene, D. (1998). UNSCATT: A visual basic software package to determine the co-ordinates of trace-length in the SCATT shooting system. Bisley, UK: GBTSF.

Gallese, V., Fadiga, L., Fogassi, L., \& Rizzolatti, G. (1996). Action recognition in the premotor cortex. Brain, 119, 593-609.

Grèzes, J., Costes, N., \& Decety, J. (1998). Top-down effect of strategy on the perception of human biological motion: A PET investigation. Cognitive Neuropsychology, 15, 553-582.

Grèzes, J., \& Decety, J. (2001). Functional anatomy of execution, mental simulation, observation, and verb generation of actions: A meta-analysis. Human Brain Mapping, 12, 1-19.

Hamzei, F., Rijntjes, M., Dettmers, C. et al. (2003). The human action recognition system and its relationship to Broca's area: An fMRI study. NeuroImage, 19, 637-644. 
Hatfield, B. D., Landers, D. M., \& Ray, W. J. (1984). Cognitive processes during self-paced motor performance: An electroencephalographic profile of skilled marksmen. Fournal of Sport Psychology, 6, 42-59.

Hedges, L. V., \& Olkin, I. (1985). Statistical methods for metaanalysis. Orlando, FL: Academic Press.

Holmes, P. S., \& Collins, D. J. (2001). The PETTLEP approach to motor imagery: A functional equivalence model for sport psychologists. Fournal of Applied Sport Psychology, 13, 60-83.

Hommel, B., Musseler, J., Aschersleben, G., \& Prinz, W. (2001). The theory of event coding: A framework for perception and action. Behavioral and Brain Science, 24, 849-878.

Iacoboni, M., Woods, R. P., Brass, M., Bekkering, H., \& Mazziotta, J. C. (1999). Cortical mechanisms of human imitation. Science, 286, 2526-2528.

Janelle, C. M., Hillman, C. H., Apparies, R. J. et al. (2000). Expertise differences in cortical activation and gaze behavior during rifle shooting. Fournal of Sport and Exercise Psychology, 22, 167-182.

Jasper, H. H. (1958). The ten-twenty electrode system of the international federation. Electroencephalography and Clinical Neurophysiology, 10, 371-375.

Jeannerod, M. (1994). The representing brain: Neural correlates of motor intention and imagery. Behavioral and Brain Sciences, $17,187-245$.

Jeannerod, M. (1999). The 25th Bartlett Lecture - To act or not to act: Perspectives on the representation of actions. Quarterly fournal of Experimental Psychology, 52, 1-29.

Jeannerod, M. (2001). Neural simulation of action: A unifying mechanism for motor cognition. NeuroImage, 14, S103-S109.

Kerick, S. E., McDowell, K., Hung, T. M., Laine Santa Maria, D., Spalding, T. W., \& Hatfield, B. D. (2001). The role of the left temporal region under the cognitve motor demands of shooting in skilled marksmen. Biological Psychology, 58, 263-277.

Konttinen, N., \& Lyytinen, H. (1992). Physiology of preparation: Brain slow waves, heart rate, and respiration preceding triggering in rifle shooting. International fournal of Sport Psychology, 23, 110-127.

Konttinen, N., Lyytinen, H., \& Viitasalo. J. (1998). Preparatory heart rate patterns in competitive rifle shooting. Fournal of Sports Sciences, 16, 235-242.

Lang, P. J. (1979). A bio-informational theory of emotional imagery. Psychophysiology, 17, 495-512.

Loze, G. M., Collins, D. J., \& Holmes, P. S. (2001). Pre-shot EEG alpha-power reactivity during expert air-pistol shooting: A comparison of best and worst shots. Fournal of Sports Sciences, $19,727-733$.
Loze, G. M., Collins, D. J., \& Shaw, J. C. (1999). EEG alpha rhythm, intention and oculomotor control. International fournal of Psychophysiology, 33, 163-167.

Oldfield, R. C. (1971). The assessment and analysis of handedness: The Edinburgh Inventory. Neurophysiology, 9, 97-113.

Pfurtscheller, G. (1999). Quantification of ERD and ERS in the time domain. In G. Pfurtscheller \& F. H. Lopes da Silva (Eds.), Handbook of electroencephalography and clinical neurophysiology (Vol. 6, pp. 89-105). Amsterdam: Elsevier.

Pfurtscheller, G., \& Lopes da Silva, F.H. (1999). Event-related EEG/MEG synchronization and desynchronization: Basic principles. Clinical Neurophysiology, 110, $1842-1857$.

Picton, T. W., \& Hillyard, S. A. (1972). Cephalic skin potentials in EEG. Electroencephalography and Clinical Neurophysiology, 33, 419-424.

Prinz, W. (1997). Perception and action planning. European fournal of Cognitive Psychology, 9, 129-154.

Regan, D. (1989). Human brain electrophysiology. Amsterdam: Elsevier.

Rizzolatti, G., Carmada, R., Fogassi, L. et al. (1988). Functional organization of inferior area 6 in the macaque monkey: II. Area F5 and the control of distal movements. Experimental Brain Research, 71, 491-507.

Rizzolatti, G., Fadiga, L., Gallese, V., \& Fogassi, L. (1996). Premotor cortex and the recognition of motor actions. Cognitive Brain Research, 3, 131-141.

Serrien, D. J., Fisher, R. J., \& Brown, P. (2003). Transient increases of synchronized neural activity during movement preparation: Influence of cognitive constraints. Experimental Brain Research, 153, 27-34.

Shaw, D. J., Holmes, P. S., Wilkerson, D. P., \& Jones, A. J. (2004). Cardio-respiratory responses during observation of heavy intensity exercise. Australian fournal of Psychology, 56, 135.

Stam, C. J., \& van Dijk, B. W. (2002). Synchronization likelihood: An unbiased measure of generalized synchronization in multivariate data sets. Physica D, 163, 236-251.

Vickers, J. N. (1996). Visual control when aiming at a far target. fournal of Experimental Psychology: Human Perception and Performance, 22, 342-354.

Williams, J. D., Rippon, G., Stone, B. M., \& Annett, J. (1995). Psychophysiological correlates of dynamic imagery. British fournal of Psychology, 86, 283-300. 